\title{
Editorial
}

\section{Years of Vitamins}

In 1912, Casimir Funk introduced the term "vitamine" for at this time still mostly unknown micronutrients that are essential for life. The wealth of knowledge that has been gathered since then was a reason enough to celebrate the $100^{\text {th }}$ anniversary in Basel, where vitamins played an important role in the scientific and industrial community.

During the first half of the $20^{\text {th }}$ century 13 molecules have been described, isolated and chemically synthetized that fulfill various functions in the metabolism know today as vitamin $\mathrm{A}, \mathrm{D}, \mathrm{E}, \mathrm{K}, \mathrm{B}_{1}, \mathrm{~B}_{2}$, $\mathrm{B}_{6}, \mathrm{~B}_{12}$, folic acid, niacin, pantothenic acid, biotin, and C. Other compounds have been proposed to have similar or complementary functions, but none of them reached the criteria of essentiality. Thus, the question arises whether we know everything about vitamins or whether we still need to fill gaps in our understanding of their functionality.
The symposium covered, therefore, the three areas past, present and future of vitamin research. At the beginning of the $20^{\text {th }}$ century, scientists had to fight against the dogma that diseases can only be caused by germs. Several decades later enthusiastic expectations of almost unlimited health benefits had to be dampened. Today, recommendations for the intake of micronutrients are revised with respect to the particular requirements of various age groups and of individual living conditions. The future will reveal whether we need to or whether we can go a step further and advice each individual according to their personal needs.

Although the lecturers of the meeting did not give an answer to all open questions, many suggestions or ideas for future research might be derived from this comprehensive compilation of the symposium.

U. Moser, SGE and I. Elmadfa, ÖGE 